\title{
Relativistic Wave Equation for Two Dirac Particles, Suggested by the Duffin-Kemmer-Petiau Algebra.
}

\author{
W. KRóLIKowSKI $\left.{ }^{*}\right)$
}

Max-Planck-Institut für Physik und Astrophysik, Werner-Heisenberg-Institut für Physik P.O. Box 401212, Munich, B.R.D.

(Nuovo Cimento A, 100, 701 (1988))

PACS 11.10.Qz - Relativistic wave equations.

On p. 707, in the caption of table II, there is $V(r)=\frac{1}{2}\left(\beta_{1}+\beta_{2}\right) S(r)$ and should be $V(r)+\frac{1}{2}\left(\beta_{1}+\beta_{2}\right) S(r)$.

On p. 711 , in the rows 6-7 from below the same error is repeated.

On p. 711, in the last equation (A.1), there is $-i \boldsymbol{p} \phi=\left(m+\frac{1}{2} S\right) \phi$ and should be $-i \boldsymbol{p}\rangle=\left(m+\frac{1}{2} S\right) \boldsymbol{\phi}$.

${ }^{*}$ ) On leave of absence from Institute of Theoretical Physics, Warsaw University, Warsaw, Poland. 